Review Article

\title{
Insights into COVID-19 Delta variant (B.1.617.2)
}

\section{Angel Yun-Kuan Thye', Ke-Yan Loo ${ }^{1}$, Kyle Bond Chene Tan ${ }^{2}$, Jenny May-Sim Lau ${ }^{3}$, Vengadesh Letchumanan ${ }^{1 *}$}

\begin{tabular}{|c|c|}
\hline Article History & $\begin{array}{l}{ }^{1} \text { Novel Bacteria and Drug Discovery (NBDD) Research Group, Microbiome } \\
\text { and Bioresource Research Strength (MBRS), Jeffrey Cheah School of }\end{array}$ \\
\hline Received: 22 September & Medicine and Health Sciences, Monash University Malaysia, Selangor Darul \\
\hline 2021; & Ehsan 47500, Malaysia; angelthye.yunkuan@monash.edu (AYKT); \\
\hline Received in Revised Form: & ke.loo@monash.edu (KYL) \\
\hline 20 October 2021; & ${ }^{2}$ Northern Ireland Centre for Stratified Medicine (NICSM), Biomedical \\
\hline Accepted: 24 October 2021; & $\begin{array}{l}\text { Sciences Research Institute, Ulster University, C-TRIC Building, Londonderry } \\
\text { BT47 6SB. United Kingdom: Tan-BC@ulster.ac.uk (KBCT) }\end{array}$ \\
\hline Available Online: 4 & ${ }^{3}$ Queen's University Belfast, Wellcome-Wolfson Institute for Experimental \\
\hline & $\begin{array}{l}\text { Medicine, } 97 \text { Lisburn Road, Belfast BT9 7BL, United Kingdom; } \\
\text { jennymf.lau@ hotmail.com (JMSL) }\end{array}$ \\
\hline & $\begin{array}{l}\text { *Corresponding author: Vengadesh Letchumanan; Novel Bacteria and Drug } \\
\text { Discovery (NBDD) Research Group, Microbiome and Bioresource Research } \\
\text { Strength (MBRS), Jeffrey Cheah School of Medicine and Health Sciences, }\end{array}$ \\
\hline & $\begin{array}{l}\text { Monash University Malaysia, Selangor } \\
\text { vengadesh.letchumanan1@ } @ \text { monash.edu (VL) }\end{array}$ \\
\hline
\end{tabular}

\begin{abstract}
Since beginning of the severe acute respiratory syndrome coronavirus 2 (SARSCoV-2), different variants of concern (VOC) have been discovered. One of the variants that stood out was the Delta variant (B.1.617.2), first found in India. It caught worldwide attention due to its greater transmissibility than the progenitor strain and the first variant of concern (VOC)- Alpha variant (B.1.1.7). B.1.617.2 spread rapidly across the globe and became a VOC due to its high transmissibility, clinical implications, and impact on vaccine efficacy. This review discusses the background and prevalence of B.1.617.2 and its sensitivity to convalescent sera and vaccinated individuals. We will provide an insight into the impact B.1.617.2 has on vaccine efficacy and discuss the level and type of protection an individual could get by being vaccinated. We will also discuss briefly on the COVID-19 vaccine booster doses and whether it is needed.
\end{abstract}

Keywords: SARS-CoV-2, Delta (B.1.617.2), variants of concern (VOC), transmissibility, vaccine, booster

\section{Introduction}

The COVID-19 global pandemic caused by the severe acute respiratory syndrome coronavirus 2 (SARS-CoV-2) that took place in 2020 has yet to come to an end ${ }^{[1-4]}$. Throughout the pandemic, the SARS-CoV-2 spike protein is continuously mutating, leading to the discovery of more and more new variants. Four of the many variants discovered have 
been listed as variants of concern (VOC). This includes variants B.1.1.7 (Alpha), B.1.351 (Beta), B.1.617.2 (Delta) and P.1 (Gamma) ${ }^{[5]}$.

B.1.617.2 was initially given the designation as a variant of interest (VOI) by the World Health Organization (WHO) in April 2021, which quickly turned into a VOC in May $2021^{[6]}$. Interestingly, as the Delta variant became the predominant lineage in many countries, its sub-lineages are given the alias AY. This separates B.1.617.2 into smaller related clusters, allowing for better monitoring of diversity and enhancing the identification of newly emerging clades. New AY lineages are phylogenetically defined and treated as Delta variants. Their designation to a sub-lineage does not necessarily imply any functional biological differences compared to B.1.617.2 unless stated otherwise by other health bodies or the $\mathrm{WHO}^{[7]}$.

On a side note, in recent months, a new variant called the Delta plus (B.1.617.2.1 and AY.1) has emerged from India and has spread to a few other countries, including the United States (US). An analysis showed that this variant traveled to the US via England and Japan, with the first case reported in Washington state on the $3^{\text {rd }}$ May 2021. Research, however, found AY.1 to be more than just a Delta variant with an additional K417N mutation, and compared to B.1.617.2, AY.1 has a distinct mutation profile ${ }^{[8]}$. This review focuses only on B.1.617.2 which gained widespread attention due to its sudden emergence, rapid transmission, and severe clinical implications. In comparison to previous circulating variants, B.1.617.2 seems to be a more virulent variant. It is deemed to have greater transmissibility, increased severity risk, and is more resistant to current vaccines. Based on the weekly epidemiological update of COVID-19 on $31^{\text {st }}$ August 2021 published by the WHO, B.1.617.2 has been reported in 170 countries $^{[9]}$. Hence, we aim to discuss the background and prevalence of B.1.617.2 and provide valuable insights into how this variant caused the crisis, planting fear in people worldwide. We will also discuss concerns regarding covid vaccines against the B.1.617.2.

\section{Background and Prevalence of B.1.617.2}

B.1.617.2 first emerged in India in December $2020^{[5]}$, causing the second wave of COVID-19 in India, which resulted in a health crisis affecting millions of people ${ }^{[10]}$. The confirmed daily new cases in India spiked from 53 per million population (up to March 2021) to $>200$ per million population (after mid-March 2021). On the contrary, the ratio of daily recoveries to daily new cases from the beginning of March declined sharply, indicating the severe burden on India's healthcare system. Shortages in essential supplies were seen in leading hospitals and the number of deaths of COVID-19 patients in intensive care increased in $>130$ cities as a result of oxygen and medicine shortage ${ }^{[11]}$. On the $1^{\text {st }}$ of May 2021, India became the first country to record $>400,000$ new COVID-19 cases within 24 hours since the pandemic ${ }^{[12]}$. B.1.617.2 quickly became the most prevalent variant in many other countries, including the United Kingdom (UK) and the US. In the UK, B.1.617.2 emerged in mid-April and later accounted for $95 \%$ of all new cases ${ }^{[13]}$, in the US, it accounted for $99 \%$ of new cases $^{[14]}$, while in Portugal, it accounted for $70 \%$ of cases in Lisbon ${ }^{[13]}$. It also leads to new 
record highs for COVID-19 cases and deaths in many Southeast Asian countries, including Malaysia, Myanmar, and Indonesia ${ }^{[15]}$.

B.1.617.2 is one of the three sub-lineage of the B.1.617 lineage ${ }^{[5]}$. It has several spike mutations, including D614G, P681R, L452R, T19R, T478K, A222V, R158G, G142D; 156157 deletion in the $\mathrm{N}$ - terminal domain (NTD) and S2 substitution D950N ${ }^{[16]}$. Regarding some of the mutations, a study found D614G is linked to higher viral loads in the respiratory tract of infected individuals, increased human host infectivity, and possibly a higher transmission efficiency to SARS-CoV-2 ${ }^{[17,18]}$. It has also been shown to strengthen cleavage efficiency due to substitution on spike conformational diversity ${ }^{[19,20]}$. P681R is in the S1-S2 furin cleavage site ${ }^{[21]}$. Similarly, mutation at the P681 position also contributes to SARSCoV-2 transmission and infection ${ }^{[22,23]}$. On the other hand, L452R and T478K present in B.1.617.2 have been associated with vaccine escape and increased transmissibility ${ }^{[24]}$. Several studies showed that the L452R increases infectivity by stabilizing the SARS-CoV-2 spike glycoprotein and human angiotensin-converting enzyme 2 (ACE2) receptor interaction ${ }^{[25-27]}$. The stronger cell-virus attachment and increased infectivity result as the L452R mutation causes huge increments in free energy at the receptor-binding domain (RBD) and ACE2 binding complex ${ }^{[26,28]}$. Other studies also demonstrated that L452R mutant could evade the human leukocyte antigen (HLA)-24 limited cellular immunity, boost viral infectivity, and possibly increase viral replication ${ }^{[29]}$. Together, these mutations contribute to the increased transmissibility, infectivity and immune evasion characteristics of B.1.617.2.

Research indicated that B.1.617.2 is $60 \%$ more transmissible than the B.1.1.7 which is already more transmissible than the progenitor strain ${ }^{[30]}$. In addition, when most countries enforced lockdown measures, the B.1.617.2 was found to have a mean basic reproductive number $(R 0)$ value of 5.08 while the ancestral strain's $R 0$ is $2.79^{[31]}$. A China study has proposed that B.1.617.2 has a higher viral replication rate, resulting in their infections having a 1000 times higher viral load than the 19A/19B strains (during the initial 2020 epidemic) infections when the day testing turns positive. Hence, greater infectivity during the early stages $^{[32]}$. A Singapore study also associated B.1.617.2 with a lower polymerase chain reaction $(\mathrm{PCR})$ cycle threshold $(\mathrm{Ct})$ value and a longer viral shedding, possibly providing a mechanism for increased transmissibility ${ }^{[33]}$. Hence, suggesting the need for a greater vaccination rate.

Furthermore, B.1.617.2 has a higher risk of hospitalization and greater disease severity than B.1.1.7 $7^{[34-36]}$. Studies from both England and Scotland were consistent that those infected with the delta variant have twice the risk of hospitalization than those infected with the alpha variant. Based on a cohort study in England where 74\% of COVID-19 patients were unvaccinated, they found that within 14 days of testing, those infected with B.1.617.2 have a higher risk of hospitalization and greater disease severity than those infected with the B.1.1.7 variant ${ }^{[36]}$. A Singapore study was comparing patients with B.1.1.7, B.1.351, and B.1.617.2 also found B.1.617.2 was associated with higher odds of oxygen requirement, intensive unit care (ICU) admission, or death ${ }^{[33]}$. A UK report also stated B.1.617.2 has a higher secondary attack rate compared to B.1.1.7 in both household and non-household 
contacts and traveler and non-traveler cases. In contrast, the 28-day case fatality for B.1.617.2 stays low at $0.1 \%$ compared to B.1.1.7, which is at $1.9 \%{ }^{[37]}$.

Recently, there are also concerns about whether children have an increased risk of getting infected with B.1.617.2. It seems that the increased cases in children were most likely because they are not yet fully vaccinated. Moreover, in a press conference, the Centers for Disease Control and Prevention (CDC) head Rochelle Walensky said although there are increased pediatric cases and increased overall cases, there was no increased severity in pediatric cases $^{[38]}$. (Table 1)

Table 1. Summary of the background and prevalence of B.1.617.2

\begin{tabular}{|c|c|c|}
\hline & Delta/ B.1.617.2 & References \\
\hline Country first detected & India & [5] \\
\hline Month+Year detected & December 2020 & [5] \\
\hline $\begin{array}{l}\text { Confirmed Daily New Cases in } \\
\text { India (up to March 2021) }\end{array}$ & $\begin{array}{l}53 \text { per million population increased to }>200 \text { per } \\
\text { million population }\end{array}$ & [11] \\
\hline $\begin{array}{l}\text { Ratio of Daily Recoveries to Daily } \\
\text { New Cases in India (from mid- } \\
\text { March 2021) }\end{array}$ & Significant reduction & [11] \\
\hline Total countries affected & 170 & [9] \\
\hline Mutations & $\begin{array}{l}\text { D614G, P681R, L452R, T19R, T478K, A222V, } \\
\text { R158G, G142D; 156-157 deletion in the N- } \\
\text { terminal domain (NTD) and S2 substitution D950N }\end{array}$ & [16] \\
\hline Transmissibility & $60 \%$ more transmissible than B.1.1.7 & [30] \\
\hline Basic reproductive number $(R 0)$ & 5.08 while ancestral strain's $R 0$ is 2.79 & [31] \\
\hline $\begin{array}{l}\text { Risk of hospitalization and } \\
\text { Disease severity }\end{array}$ & $\begin{array}{l}\text { Twice the risk of hospitalization and a greater } \\
\text { disease severity compared to B.1.1.7 }\end{array}$ & {$[36]$} \\
\hline Secondary attack rate & $\begin{array}{l}\text { Higher than B.1.1.7 in both household and non- } \\
\text { household contacts and traveller and non-traveller } \\
\text { cases }\end{array}$ & {$[37]$} \\
\hline Are children at an increased risk? & Unlikely. Probably due to being unvaccinated & [38] \\
\hline
\end{tabular}

\section{Sensitivity of B.1.617.2 to Convalescent Sera and Vaccinated Individuals}

In a month 12 cohort study in France, individuals vaccinated with a single dose of either Pfizer, AstraZeneca, and Moderna vaccines demonstrated greater neutralizing antibody (Nab) titers against Alpha, Beta, and Delta variants than those convalescents of unvaccinated individuals. Hence, a single dose of vaccine boosts cross-neutralizing antibody responses to the Delta variant. However, those vaccinated with a single dose of Pfizer or AstraZeneca showed low efficiency against Delta variant with the percentage of sera of individuals vaccinated with Pfizer or AstraZeneca neutralizing B.1.617.2 to be $13 \%$ and $9 \%$ respectively. An efficient neutralizing response was generated only after the second dose ${ }^{[39]}$. An India study also showed that relative to the prototype strain (D614G), there is a 3.2 (two dose)-4.5 (one dose) fold reduction in NAb titer in the sera of Covishield vaccinees against the B.1.617.2, indicating the significance of a second dose. Moreover, it also showed that the NAbs in breakthrough participants and COVID-19 recovered individuals with one or two 
vaccine doses showed higher protection against B.1.617.2 than vaccinees who received one or two vaccine doses ${ }^{[10]}$. Another study also evaluated the neutralization of sera collected from COVID-19 recovered patients (post 5-20 weeks of infection) and individuals (post 28 days) vaccinated with two doses of Bharat Biotech's Covaxin (BBV152) against B.1.617.2. Findings demonstrated a 4.6-fold and 2.7-fold reduction in neutralization titer compared to the B.1 strain (D614G) with sera of COVID-19 recovered patients and Covaxin vaccinees. Studies have shown that against B.1.617.2, although there is a decrease in neutralization titer with Covaxin vaccinees sera, the neutralization potential is yet to be established. The broad epitope coverage in an inactivated vaccine (BBV152) reduces the magnitude of decreased neutralization against emerging variants by inducing an immune response against whole virion $^{[40]}$.

Besides that, studies have demonstrated the effectiveness of two shots of Pfizer vaccine against B.1.617.2 infection ${ }^{[41,42]}$. One study showed two shots of Pfizer vaccine demonstrated $88 \%$ efficacy two weeks after the second dose, while two shots of AstraZeneca vaccine could provide $60 \%$ protection against symptomatic disease from B.1.617.2 $2^{[43]}$. Therefore, although B.1.617.2 has demonstrated immune evasion characteristics, vaccines still offer protections against B.1.617.2 to some extent.

\section{Understanding the B.1.617.2 Variant}

There is an association between the emergence of new variants and epidemic severity and SARS-CoV-2 transmission. The high transmission rate is a prognostic factor for genomic variations. As a consequence, outbreaks happen following large gatherings ${ }^{[5,44]}$. The emergence of the B.1.617.2 has been raising concerns globally due to its high transmissibility, increased disease severity, and immune evasion ability. With the emergence of the COVID-19 pandemic, several types of vaccines have been developed, consisting of inactivated virus vaccines, a protein subunit vaccine, mRNA vaccines, and viral vector recombinant vaccines ${ }^{[45]}$. The COVID-19 vaccines enable the activation of the immune response upon binding to the spike protein ${ }^{[5]}$. However, mutations on the RBD that are present in many new emerging variants are responsible for most of the escape from vaccineinduced neutralization ${ }^{[46]}$. Thus, concerns on vaccine efficacy arise as new variants with mutations on the spike protein emerge. In addition, the durability of antibody response in semi and fully vaccinated individuals are not yet fully understood.

The toll B.1.617.2 takes depends mainly on the population's vaccination status and the number of people having immunity from previous infection ${ }^{[13]}$. For instance, in the UK with a high vaccination rate, fatality did not increase with increased cases. However, in countries with low vaccination rates such as Thailand and Indonesia, the COVID-19 cases are more serious. New cases in the US also were focused on unvaccinated individuals ${ }^{[47]}$. Another example is in Israel, the estimated Pfizer vaccine effectiveness against SARS-CoV2 infection was $95.3 \% \geq 7$ days after the second dose ${ }^{[48]}$. However, the Israeli Ministry of Health stated that the efficacy of the Pfizer vaccine dropped to 39\% due to B.1.617.2. Even so, there is still $88 \%$ protection against infection, progressing to hospitalization, and $94.1 \%$ 
against severe illness $^{[47]}$. Thus, demonstrating vaccination still plays a role in alleviating the disease burden.

According to data from the Coronavirus Resource Center, John Hopkins University of Medicine, many countries have initiated a national vaccination process. Many have indeed had a high percentage of the population vaccinated. However, there are still a handful of countries whose vaccination rate is still far below the world average ${ }^{[49]}$. Not to forget, as mentioned earlier, B.1.617.2 has a much higher reproduction number compared to the ancestral strain, which means a much higher vaccine coverage needs to be achieved compared to the assumed initially 60-70\% vaccine coverage for the ancestral strain. A reproductive number of 5 would mean a vaccine coverage of $80 \%$ will be needed based on the equation $\mathrm{q}=1-1 / R 0$, assuming a $100 \%$ vaccine efficacy ${ }^{[31]}$. B.1.617.2, having high infectivity means more people need to be vaccinated to reduce its spread and disease burden. Hence, it is crucial to increase vaccination coverage to control the spread B.1.617.2.

Now, for some countries where most of the population had been fully vaccinated with two doses of COVID-19 vaccine, this brings us to the issue of COVID-19 vaccine booster doses. Currently, the CDC and the Food and Drug Administration (FDA) have approved the booster shot of the Pfizer-BioNTech COVID-19 vaccine in specific populations and those in high-risk occupational and institutional settings, while booster shots of COVID-19 vaccines by Moderna and Johnson \& Johnson will be evaluated in the coming weeks. According to the statement released by the CDC on $24^{\text {th }}$ September 2021, with the dominance of B.1.617.2 and the increase in COVID-19 cases in the US, booster shots can strengthen protection against severe disease in those with high-risk exposure to COVID-19 or complications for severe disease ${ }^{[50]}$. With that, Israel is the first country in the world to administer Covid-19 vaccine (Pfizer) booster doses to adults with weak immune systems following the announcement by the Israeli Ministry of Health on the $11^{\text {th }}$ July $2021^{[47]}$.

However, some believe booster doses should not be prioritized over primary vaccination. The clinical trials lead for the vaccine and director of the Oxford Vaccine Group, Andrew Pollard emphasized "urgent priority" should be given to those who have not got their first dose before initiating a third booster dose ${ }^{[51]}$. In line with that, a statement released by the WHO on the $4^{\text {th }}$ August stated that 4 billion vaccine doses had been administered globally, in which $>80 \%$ went to upper-middle- and high-income countries even though they accounted for $<50 \%$ of the world's population. Although booster doses may help combat the Delta variant, people from low-income countries should not be left unprotected ${ }^{[52,53]}$. Moreover, current evidence is limited and inconclusive on the widespread need for booster doses after receiving primary vaccination ${ }^{[54]}$.

\section{Conclusion}

Despite the concerns mentioned regarding the B.1.617.2, national and international vaccination is still crucial for containing this global SARS-CoV-2 pandemic, as vaccines induce neutralizing antibodies and block the viral RBD from binding to the ACE2 receptor. Although there is a reduction in vaccine efficacy against the B.1.617.2, vaccines can still 
provide a considerable amount of protection, decreasing the risk of hospitalization and moderating the severity ${ }^{[5]}$. Nonetheless, the government should implement testing campaigns, mass vaccination, efficient contact-tracing, restricting gatherings, have strict quarantine of international travelers and strict traveling bans ${ }^{[55-58]}$. Furthermore, reminders and awareness within the community could increase compliance to preventive measures ${ }^{[59]}$. With the continuous emergence of new variants, there is a need for constant surveillance of the evolutionary changes of SARS-CoV-2 and how mutations of the spike protein contribute to immune escape ${ }^{[60]}$. This could allow for better definition and implementation of countermeasures. Last but not least, we must continue practicing safety measures to protect ourselves and others from COVID-19 infection and slow its transmission ${ }^{[5,61]}$.

Author Contributions: AY-KT performed the literature search, critical data analysis as well as manuscript writing. K-YL, KBCT, JM-SL, and VL performed technical support and proofreading of this writing. VL provided conceptualization and set up this review writing project. All authors have read and agreed to the published version of the manuscript.

Funding: This work is supported by Early Career Research Grant, Jeffrey Cheah School of Medicine and Health Sciences ECR-000021, awarded to VL.

Conflicts of Interest: The authors declare no conflict of interest.

\section{References}

1. Goh HP, Mahari WI, Ahad NI, et al., Risk factors affecting COVID-19 case fatality rate: A quantitative analysis of top 50 affected countries. Prog Microbes Mol Biol 2020; 3(1).

2. Letchumanan V, Ab Mutalib N-S, Goh B-H, et al., Novel coronavirus 2019-nCoV: Could this virus become a possible global pandemic. Prog Microbes Mol Biol 2020; 3(1).

3. Loh HC, Seah YK, and Looi I, The COVID-19 pandemic and diet change. Prog Microbes Mol Biol Biology 2021; 4(1).

4. Lee VS, Chong WL, Sukumaran SD, et al., Computational screening and identifying binding interaction of anti-viral and anti-malarial drugs: Toward the potential cure for SARS-CoV-2. Prog Drug Discovery Biomed Sci 2020; 3(1).

5. Thye AY-K, Law JW-F, Pusparajah P, et al., Emerging SARS-CoV-2 Variants of Concern (VOCs): An Impending Global Crisis. Biomedicines 2021; 9(10): 1303.

6. Tracking SARS-CoV-2 variants. $202122^{\text {nd }}$ September 2021 [Accessed 2021 Sep 3]; Available from: https://www.who.int/en/activities/tracking-SARS-CoV-2-variants/.

7. SARS-CoV-2 variants of concern and variants under investigation in England. Technical briefing 23. 2021: Public Health England.

8. Kannan SR, Spratt AN, Cohen AR, et al., Evolutionary analysis of the Delta and Delta Plus variants of the SARS-CoV-2 viruses. J Autoimmunity 2021; 124: 102715.

9. Weekly epidemiological update on COVID-19 - 31 August 2021. $202131^{\text {st }}$ August 2021 [Accessed 2021 Sep 3]; 55:[Available from: https://www.who.int/publications/m/item/weekly-epidemiologicalupdate-on-covid-19---31-august-2021.

10. Sapkal GN, Yadav PD, Sahay RR, et al., Neutralization of Delta variant with sera of Covishield ${ }^{\mathrm{TM}}$ vaccinees and COVID-19-recovered vaccinated individuals. J Travel Med 2021.

11. Singh J, Rahman SA, Ehtesham NZ, et al., SARS-CoV-2 variants of concern are emerging in India. Nat Med 2021: 1-3.

12. Joseph RJ and Ser H-L, Stories from the East: COVID-19 Situation in India. Prog Microbes Mol Biol 2021; 4(1).

13. Vaughan A, Delta to dominate world. 2021, Elsevier. 
14. Dyer O, Covid-19: Unvaccinated face 11 times risk of death from delta variant, CDC data show. 2021, British Medical Journal Publishing Group.

15. Kwan JN, Loh HC, and Looi I, COVID-19 Vaccination during Pregnancy in Southeast Asia. Prog Microbes Mol Biol 2021; 4(1).

16. Liu C, Ginn HM, Dejnirattisai W, et al., Reduced neutralization of SARS-CoV-2 B. 1.617 by vaccine and convalescent serum. Cell 2021; 184(16): 4220-4236. e13.

17. Korber B, Fischer WM, Gnanakaran S, et al., Tracking changes in SARS-CoV-2 spike: evidence that D614G increases infectivity of the COVID-19 virus. Cell 2020; 182(4): 812-827. e19.

18. Volz E, Hill V, McCrone JT, et al., Evaluating the effects of SARS-CoV-2 spike mutation D614G on transmissibility and pathogenicity. Cell 2021; 184(1): 64-75. e11.

19. Kim YJ, Jang US, Soh SM, et al., The Impact on Infectivity and Neutralization Efficiency of SARSCoV-2 Lineage B. 1.351 Pseudovirus. Viruses 2021; 13(4): 633.

20. Gobeil SM-C, Janowska K, McDowell S, et al., D614G mutation alters SARS-CoV-2 spike conformation and enhances protease cleavage at the S1/S2 junction. Cell Rep 2021; 34(2): 108630.

21. Cherian S, Potdar V, Jadhav S, et al., SARS-CoV-2 Spike Mutations, L452R, T478K, E484Q and P681R, in the Second Wave of COVID-19 in Maharashtra, India. Microorganisms 2021; 9(7): 1542.

22. Peacock TP, Goldhill DH, Zhou J, et al., The furin cleavage site in the SARS-CoV-2 spike protein is required for transmission in ferrets. Nat Microb 2021: 1-11.

23. Hoffmann M, Kleine-Weber H, and Pöhlmann S, A multibasic cleavage site in the spike protein of SARS-CoV-2 is essential for infection of human lung cells. Mol Cell 2020; 78(4): 779-784. e5.

24. Adam D, What scientists know about new, fast-spreading coronavirus variants. Nat 2021.

25. Deng X, Garcia-Knight MA, Khalid MM, et al., Transmission, infectivity, and neutralization of a spike L452R SARS-CoV-2 variant. Cell 2021.

26. Chen J, Wang R, Wang M, et al., Mutations strengthened SARS-CoV-2 infectivity. J Mol Biol 2020; 432(19): 5212-5226.

27. Teng S, Sobitan A, Rhoades R, et al., Systemic effects of missense mutations on SARS-CoV-2 spike glycoprotein stability and receptor-binding affinity. Briefings Bioinf 2021; 22(2): 1239-1253.

28. Tchesnokova V, Kulakesara H, Larson L, et al., Acquisition of the L452R mutation in the ACE2binding interface of Spike protein triggers recent massive expansion of SARS-Cov-2 variants. BioRxiv 2021.

29. Motozono C, Toyoda M, Zahradnik J, et al., SARS-CoV-2 spike L452R variant evades cellular immunity and increases infectivity. Cell Host Microb 2021; 29(7): 1124-1136. e11.

30. Mahase E, Delta variant: What is happening with transmission, hospital admissions, and restrictions? 2021, British Medical Journal Publishing Group.

31. Liu Y and Rocklöv J, The reproductive number of the Delta variant of SARS-CoV-2 is far higher compared to the ancestral SARS-CoV-2 virus. J Travel Med 2021.

32. Li B, Deng A, Li K, et al., Viral infection and transmission in a large well-traced outbreak caused by the Delta SARS-CoV-2 variant. MedRxiv 2021.

33. Ong SWX, Chiew CJ, Ang LW, et al., Clinical and Virological Features of SARS-CoV-2 Variants of Concern: A Retrospective Cohort Study Comparing B. 1.1. 7 (Alpha), B. 1.315 (Beta), and B. 1.617. 2 (Delta). 2021.

34. SARS-CoV-2 variants of concern and variants under investigation in England. Technical briefing 14. 2021: Public Health England.

35. Sheikh A, McMenamin J, Taylor B, et al., SARS-CoV-2 Delta VOC in Scotland: demographics, risk of hospital admission, and vaccine effectiveness. The Lancet 2021.

36. Twohig KA, Nyberg T, Zaidi A, et al., Hospital admission and emergency care attendance risk for SARS-CoV-2 delta (B. 1.617. 2) compared with alpha (B. 1.1. 7) variants of concern: A cohort study. The Lancet Infectious Diseases 2021.

37. SARS-CoV-2 variants of concern and variants under investigation in England. Technical briefing 15. 2021: Public Health England. 
38. Wilson C, Is delta a bigger threat to children? 2021, Elsevier.

39. Planas D, Veyer D, Baidaliuk A, et al., Reduced sensitivity of SARS-CoV-2 variant Delta to antibody neutralization. Nat 2021; 596(7871): 276-280.

40. Yadav PD, Sapkal GN, Ella R, et al., Neutralization of Beta and Delta variant with sera of COVID-19 recovered cases and vaccinees of inactivated COVID-19 vaccine BBV152/Covaxin. J Travel Med 2021.

41. Wall EC, Wu M, Harvey R, et al., Neutralising antibody activity against SARS-CoV-2 VOCs B. 1.617. 2 and B. 1.351 by BNT162b2 vaccination. The Lancet 2021; 397(10292): 2331-2333.

42. Liu J, Liu Y, Xia H, et al., BNT162b2-elicited neutralization of B. 1.617 and other SARS-CoV-2 variants. Nat 2021; 596(7871): 273-275.

43. Vaccines highly effective against B.1.617.2 variant after 2 doses. 2021: Public Health England.

44. Bandoy DDR and Weimer BC, Analysis of SARS-CoV-2 genomic epidemiology reveals disease transmission coupled to variant emergence and allelic variation. Sci Rep 2021; 11(1): 1-12.

45. Loo K-Y, Letchumanan V, Ser H-L, et al., COVID-19: Insights into potential vaccines. Microorganisms 2021; 9(3): 605.

46. Garcia-Beltran WF, Lam EC, Denis KS, et al., Multiple SARS-CoV-2 variants escape neutralization by vaccine-induced humoral immunity. Cell 2021; 184(9): 2372-2383. e9.

47. Bian L, Gao Q, Gao F, et al., Impact of the Delta variant on vaccine efficacy and response strategies. Expert Review of Vaccines 2021(just-accepted).

48. Haas EJ, Angulo FJ, McLaughlin JM, et al., Impact and effectiveness of mRNA BNT162b2 vaccine against SARS-CoV-2 infections and COVID-19 cases, hospitalisations, and deaths following a nationwide vaccination campaign in Israel: an observational study using national surveillance data. The Lancet 2021; 397(10287): 1819-1829.

49. Understanding Vaccination Progress. 2021 [Accessed 2021 Sep 3]; Available from: https://coronavirus.jhu.edu/vaccines/international.

50. CDC Statement on ACIP Booster Recommendations. 2021 [Accessed 2021 Sep 25]; Available from: https://www.cdc.gov/media/releases/2021/p0924-booster-recommendations-.html.

51. Mahase E, Covid-19: Third vaccine dose boosts immune response but may not be needed, say researchers. 2021, British Medical Journal Publishing Group.

52. Mahase E, Covid-19 booster vaccines: What we know and who's doing what. 2021, British Medical Journal Publishing Group.

53. WHO Director-General's opening remarks at the media briefing on COVID-19-4 August 2021.2021 [Accessed 2021 Sep 3]; Available from: https://www.who.int/director-general/speeches/detail/whodirector-general-s-opening-remarks-at-the-media-briefing-on-covid-4-august-2021.

54. Interim statement on booster doses for COVID-19 vaccination. $20214^{\text {th }}$ October 2021 [Accessed 2021 Oct 10]; Available from: https://www.who.int/news/item/04-10-2021-interim-statement-on-boosterdoses-for-covid-19-vaccination.

55. Rahimi F and Abadi ATB, Emergence of the Delta Plus variant of SARS-CoV-2 in Iran. Gene Rep 2021.

56. Ser H-L, Letchumanan V, Law JW-F, et al., PMMB COVID-19 Bulletin: Spain (18 ${ }^{\text {th }}$ April 2020). Prog Microbes Mol Biol 2020; 3(1).

57. Tan LT-H, Letchumanan V, Ser H-L, et al., PMMB COVID-19 Bulletin: United Kingdom (22 ${ }^{\text {nd }}$ April 2020). Prog Microbes Mol Biol 2020; 3(1).

58. Loo K-Y and Letchumanan V, COVID-19: Malaysia's fight against this deadly virus. Prog Microbes Mol Biol 2021; 4(1).

59. Johnson D, Ren SEC, Johnson HD, et al., COVID-19: Are Malaysians embracing or suffering the new normality? Prog Microbes Mol Biol; 3(1).

60. Ser H-L, Tan LT-H, Law JW-F, et al., Genomic analysis of severe acute respiratory syndrome coronavirus 2 (SARS-CoV-2) strains isolated in Malaysia. Prog Microbes Mol Biol 2020; 3(1). 
61. Hoo HE, Loh HC, Ch'ng ASH, et al., Positive impacts of the COVID-19 pandemic and public health measures on healthcare. Prog Microbes Mol Biol 2021; 4(1).

Author(s) shall retain the copyright of their work and grant the Journal/Publisher right for the first publication with the work simultaneously licensed under:

Creative Commons Attribution-NonCommercial 4.0 International (CC BY-NC 4.0). This license allows for the copying, distribution and transmission of the work, provided the correct attribution of the original creator is stated. Adaptation and remixing are also permitted. 